\title{
An individual-based model for Salmonella transmission along the pig production chain
}

\author{
Stefanie Hotes, Imke Traulsen and Joachim Krieter \\ Institute of Animal Breeding and Husbandry, Christian-Albrechts-Universität zu Kiel, Kiel, Germany
}

\begin{abstract}
The aim was to develop an individual-based model for the transmission of Salmonella from farrowing farm to slaughterhouse. The present study concentrated on farrowing sows as the initial source of Salmonella transmission. The model was evaluated with a sensitivity analysis using a Plackett-Burman design. Three levels (minimum, default, maximum values) for all input factors were considered. The deviations from the default prevalences caused by the extreme values did not balance each other for several significant input factors. For these factors, the relation between input factor and regarded health states was not linear.

Results showed that the probability of effective contact, to restart shedding, the shedding duration and the sow herd prevalences as well as their distribution across farrowing farms determined the Salmonella prevalence at slaughter. The study emphasised the importance of vertical and horizontal transmission. Low Salmonella prevalences obtained after nursing caused prevalences at slaughterhouse up to $12 \%$.
\end{abstract}

Keywords: stochastic modelling, Salmonella, pig production, Plackett-Burman design

\section{Introduction}

Salmonellosis is a major problem in most countries in the world (WHO 2007). The World Health Organization (WHO) has reported that diseases of zoonotic origin are of particular concern in the European Union (EU). Recent estimates predict that temperature-sensitive infectious disease, such as food-borne infections, will become more important in the coming decades (WHO 2010). Frequent cases of human salmonellosis in Germany have induced the Federal Institute for Risk Assessment (BfR) to emphasise the avoidance of raw meat, in particular raw pork in any form (BfR 2005). Salmonella prevalence in slaughter pigs in the EU is estimated at about $10 \%$. Country prevalences range from no positive findings in Finland up to a prevalence of $29 \%$ in Spanish slaughter pigs (EFSA 2008). The 2003 European regulation for the control of Salmonella and other specified food-borne zoonotic agents (EC No 2160/2003) regulates that national control programmes have to be established to provide the detection of zoonosis and specific control measures. The German implementation focuses on the finishing stage. Only if prevalence at slaughter age exceeds $40 \%$, the pigs purchased from farrowing stage should be tested as well. However, pig deliveries are certainly critical points for dynamics of Salmonella transmission. Bacteria can spread across farms due to the enhanced contact structure. Previously published models have concentrated on the spread within a single farm (Ivanek et al. 2004, Hill et al. 2008, 
Lurette et al. 2008) but have not considered the production and contact structures in detail (van der Gaag et al. 2004, Wehebrink et al. 2007).

The objective of the present study was to develop an individual-based model for the transmission of non-clinical Salmonellosis within the pork supply chain. Modelling was to reveal the most important factors for Salmonella spread with regard to the trade relationships between the production stages. The current paper represents a further development of the simulation study developed by Krieter (2004).

\section{Material and methods}

The developed simulation model describes the spread of non-clinical Salmonella infection (henceforth referred to as »infection«) within the pork supply chain from farrowing stage to slaughter. The model is stochastic with a discrete time step of one week. Comparable to all-in all-out production, the one-week time step is defined as the basic unit for regrouping tasks and moving pigs between branches of production. Only transports and processes at slaughterhouses are detached from the one-week rhythm of the model. The present study focused on the transmission of Salmonella from sow to pig and subsequently from pig to pig. The epidemiological process in the model describes the spread of Salmonella over a 52-week period with a prior burn-in also of 52 weeks. The burn-in preceded the simulation in order to initiate the trade relationships and to fill the model with pigs. At starting point, the model contains only farms and sows because output data has to be based on slaughter pigs whose whole lives were to be monitored. Without burn-in, the first pigs would reach slaughter age at week 28. The quantity and quality of the output data would be low. The burn-in ensures a continuous flow of slaughter pigs and thereby improves the results. The program is written in the object-oriented language C++. Routines from the NAG C library (NAG 2001) are used to generate random numbers. Random numbers are drawn to determine farm characteristics and trade relationships as well as individual infection and course of disease as described below.

\section{Trade relationships}

The model contains a network of farrowing and finishing farms as well as a slaughterhouse which are linked by pig deliveries. The model considers two types of farrowing farms:

1) conventional farrowing farms with supply relationships $\left(\mathrm{PP}_{\mathrm{s}}\right)$ to one $\left(\mathrm{PP}_{\mathrm{s}}\right.$ one), two $\left(\mathrm{PP}_{\mathrm{s}-}\right.$ two) or three finishing farms ( $\mathrm{PP}_{\mathrm{s}-}$ three);

2) farrow-to-finishing farms without supply relationships to a specialised finishing farm $\left(P_{\mathrm{F}}\right)$.

At the finishing stage, fatteners buying pigs from one ( $F \_$one), two (F_two) or three (F_three) conventional farrowing farms are distinguished. Figure 1 illustrates several possible trade relationships. The only information obligatory for the network concerns the farrowing farms and contains the sow herd size (varying from 100 to 350 sows with a mean of 181 sows in the current study), the suckling period (four weeks by default for the current study) and the production cycle (mix of one and three weeks in the current study). Prior to every replication a regeneration of the trade relationships is performed considering the stated distributions (Table 1, no. 1-3). The regeneration implicates the classification of the farrowing farms into 


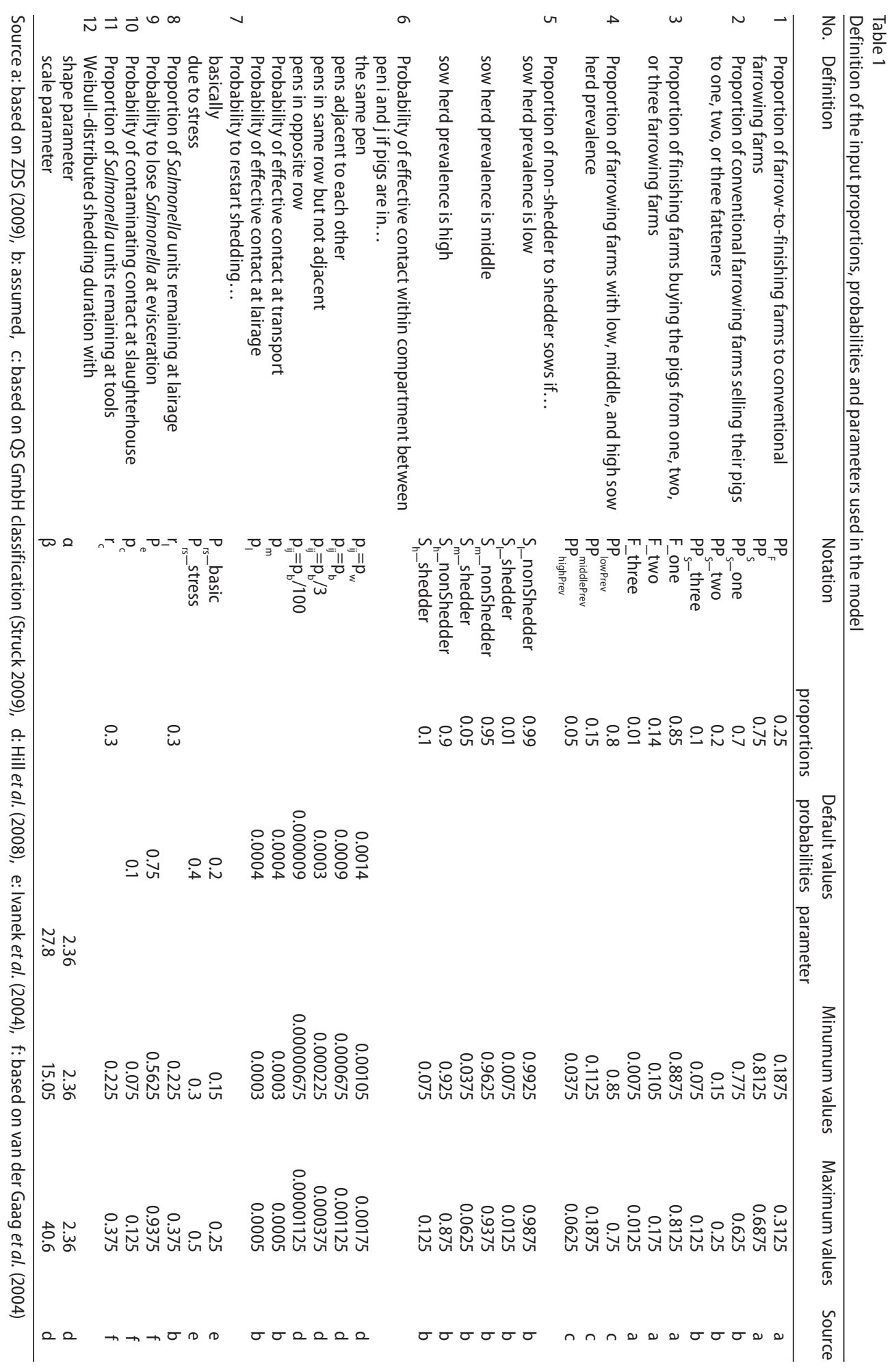


farms with a low $\left(\mathrm{PP}_{\text {lowPrev }}\right)$, middle $\left(\mathrm{PP}_{\text {middlePrev }}\right)$, or high sow herd prevalence level ( $\left.\mathrm{PP}_{\text {highPrev }}\right)$. Based on this classification each sow on the farm has a certain probability to become a shedder (S_shedder) or non-shedder (S_nonShedder) (Table 1; no. 4 and 5). The health states of the sows are not regarded in more detail and remain unchanged for the respective replication.

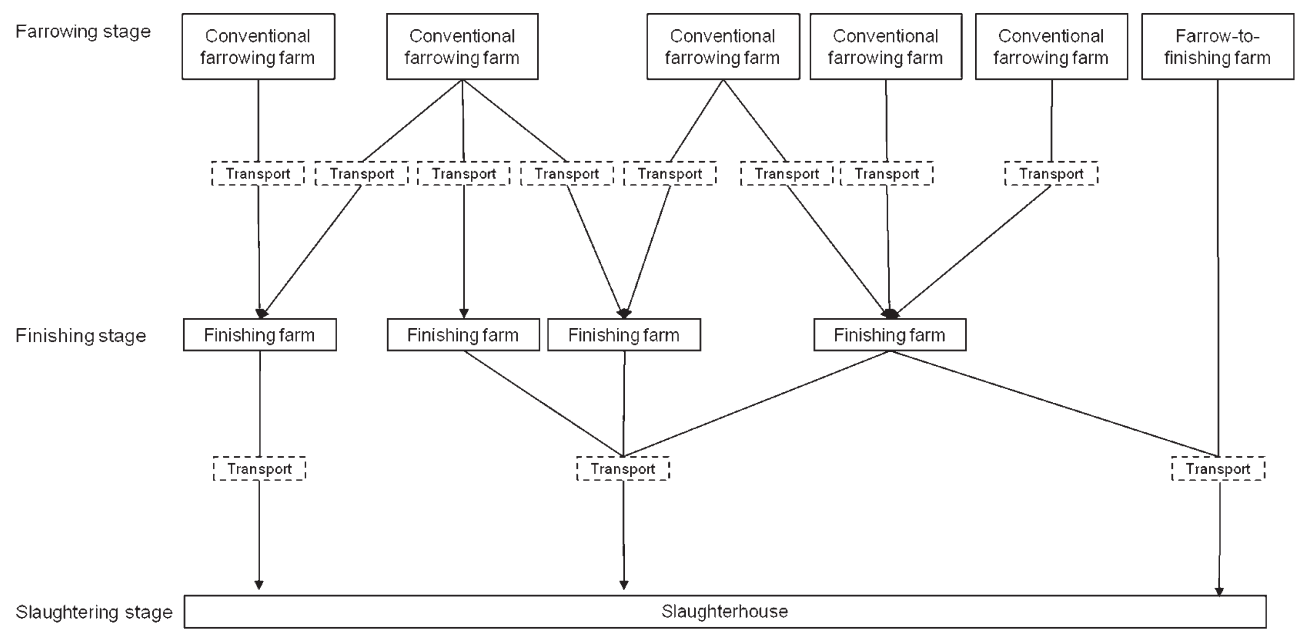

Figure 1

Illustration of possible trade relationships within the simulated pork supply chain

\section{Health and contamination states}

The program is individual-based i.e. each pig is followed from farrowing to chilling at slaughter. During its lifetime the pig's health status is updated every week. As described by Lurette et al. (2008), four mutually exclusive health states are distinguished: susceptible pigs free of Salmonella and thus non-shedding and seronegative (S-); seronegative, shedding pigs (I-); seropositive, shedding pigs (I+), and seropositive non-shedding but Salmonellabearing pigs $(\mathrm{C}+$ ) (Figure 2). The latent period between Salmonella ingestion and shedding falls below the model basis of one week (Lurette et al. 2008) and is therefore neglected. The seroconversion is assumed to last about two weeks (van der Gaag et al. 2004). Subsequent to seroconversion, the pig stays seropositive. The possibility of recovery is not considered. Even if a return to seronegativity should exist in reality, it is assumed to last longer than the lifetime of the pigs (Lurette et al. 2008). The only change in health state based on the carrier stage is to restart shedding and become I+ again. The risk that the carrier pigs restart shedding is always present $\left(\mathrm{P}_{\mathrm{rs}}\right.$-basic) but increases if the pigs are exposed to stress $\left(\mathrm{P}_{\mathrm{rs}-}\right.$ stress) (Table $1 ;$ no. 7). Whether it is the first shedding period (I- until $C+$ ) or a subsequent one (starting from $\mathrm{C}+\mathrm{I}$ I+ to $\mathrm{C}+$ ), the shedding duration is assumed to be Weibull-distributed (Hill et al. 2008) with a mean shedding duration of about four weeks (Table 1, no. 12). All piglets are born susceptible to infection and infection is immediately possible within the farrowing crate. The aspect of passive immunity of newborn piglets from a sow's antibodies is however neglected, due to the lack of precise information. 
The health state of the pig does not change post-slaughter (Figure 2). But slaughter pigs lose bacteria due to careful evisceration or become contaminated on their skin surfaces due to the slaughter sequence (direct contact of carcasses) or contaminated tools (cross-contamination) (Table 1; nos 8-11).

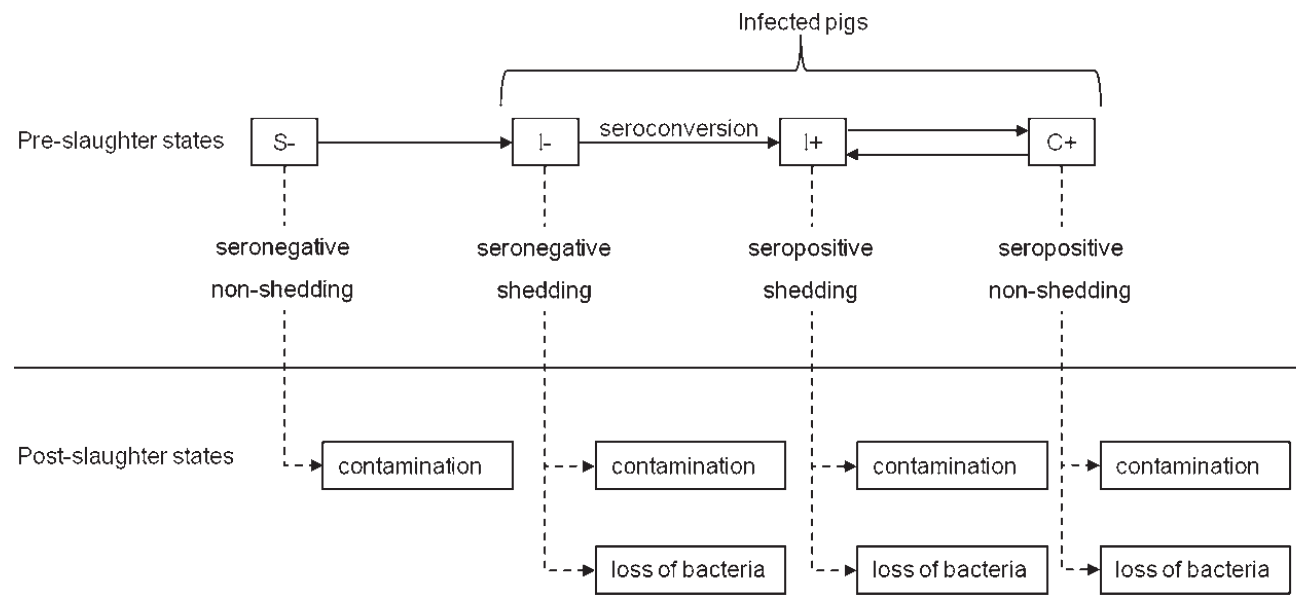

Figure 2

Health and contamination states of pigs pre- and post-slaughter

\section{Probability of infection and contamination}

The modelling of infection is based on the Reed-Frost epidemic model, which describes the probability $P(t)$ of a susceptible pig having effective contact with any of $E(t)$ excreting pigs during the period $[t-1, t]$ (Rubel 2005, Hill et al. 2008).

$$
P(t)=1-(1-p)^{E(t)}
$$

Effective contact is defined as contact between an infectious individual and a susceptible one, which produces a newly infected individual (Bailey 1975). The probability for an effective contact is given by $p$.

The current program considers the sows of the farrowing farms as the initial sources of Salmonella infection. Consequently, Salmonella is transmitted only from sow to pig and subsequently through transmission from pig to pig without Salmonella entry via rodents, birds, feed et cetera. In the following, the possibilities for effective contact between two individuals are described in detail.

\section{Infection at farm}

Assuming that all pigs housed in pens of the same compartment have contact with each other, the probability of an effective contact increases with an increasing number of shedding pigs. Contact can happen via faecal or airborne transmission. Formula 2 describes the probability $P_{i j}$ of a susceptible pig in pen $i$ becoming infected because of $E_{j}(t)$ excreting pigs in pen $j$ during the period [t-1, $\mathrm{t}$ ] (Hill et al. 2008): 


$$
P_{i j}(t)=1-\left(1-p_{i j}\right)^{E_{j}(t)}
$$

The probability of effective contact between pens i and $j\left(p_{i j}\right)$ depends on the distance between these pens (Table 1; no. 6). The number of pens within a compartment varies between farms but all compartments consist of two opposite rows. During nursing, transmission is assumed to be reduced to pen level. This assumption is based on the limited contacting of new born piglets to piglets of other pens.

\section{Infection at transport}

In an agglomerated pig producing area such as Northern Germany, transport from farrowing farm to finishing farm does not exceed a few hours. Precise data about new infections during transport are missing. Pigs are stressed due to transport and prior fasting whereby carrier pigs could restart shedding. This is implemented in the program by an increased probability for shedding reactivation ( $\mathrm{P}_{\mathrm{rs}-}$ stress; Table $1 ;$ no. 7$)$. Hence, in the model, the increased number of shedding pigs increases the probability of a susceptible pig becoming infected at the fattening compartment $\left(\mathrm{P}_{\mathrm{ij}}\right)$. This mainly compensates for the missing infection opportunity during transport to the finishing stage.

In contrast, transport to slaughterhouse is not followed by an extensive housing period. But transport to slaughterhouse lasts much longer compared to transport to finishing farm, which increases the probability of effective contact at the lorry $\left(p_{m}\right.$; Table $1 ;$ no. 6). The probability $P_{m}$ of a susceptible pig becoming infected due to effective contact with any of $E_{m}(t)$ excreting pigs at transport to slaughterhouse is represented by:

$$
P_{m}(t)=1-\left(1-p_{m}\right)^{E_{m}(t)}
$$

An increased probability of shedding restart is also considered at transport to slaughterhouse (Table 1; no. 7).

\section{Infection at lairage}

After transport to the slaughterhouse, the pigs are housed at lairage until the slaughter process starts. Comparable to infection at the farm and during transport the probability of infection at lairage ( $p_{j}$; Table 1 ; no. 6$)$ increases with an increasing number of Salmonellashedding pigs in the proximity. The lairage is neither cleaned intensively nor disinfected before a new group of pigs is housed. Comprehensive cleaning is only possible at the end of a working day. Insufficient cleaning during the day increases the probability of infection at lairage. In addition to the number of shedding pigs in the current group $\left(\mathrm{E}_{\mathrm{I}}(\mathrm{t})\right)$, the Salmonella output of all previous groups has to be considered $\left(R_{1}\right)$.

$$
P_{\text {I }}(t)=1-(1-p)^{E_{l}(t)+R_{I}(t)}
$$

with $R_{l}(t)=\left(E_{l}(t-1)+R_{l}(t-1)\right) \times r_{l}$

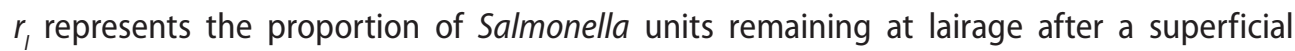
cleaning (Table 1; no. 8). 
Loss of bacteria and contamination at slaughter line

After death, the pigs are no longer able to change their health state, however surface contamination and loss of bacteria is possible. The probability of losing Salmonella $\left(\mathrm{P}_{\mathrm{e}}\right)$ depends on how carefully evisceration is performed (Table 1; no. 9). Carcass contamination could happen via direct contact of carcasses or cross-contamination due to soiled equipment and tools. Whether a pig becomes contaminated at the slaughter process depends preliminarily on the probability of a contaminating contact $\left(p_{c}\right.$; Table $1 ;$ no. 10$)$ and the Salmonella status of the previously slaughtered pigs $\left(E_{c}(t)\right)$. Furthermore, pigs already slaughtered could have contaminated the equipment and tools whereby bacteria could skip carcasses or contaminate a whole array of subsequent carcasses.

$$
\begin{aligned}
P_{c}(t) & =1-\left(1-p_{c}\right)^{E_{c}(t)+R_{c}(t)} \\
\text { with } E_{c}(t) & =\left\{\begin{array}{l}
0 \text { if Salmonella status of previous slaughtered pig }=S- \\
1 \text { if Salmonella status of previous slaughtered pig }=1-, 1+\text { or } C+
\end{array}\right. \\
R_{c}(t) & =\left(E_{c}(t-1)+R_{c}(t-1)\right) \times r_{c}
\end{aligned}
$$

$R_{c}$ describes the impact of all previously slaughtered pigs with $r_{c}$ representing the proportion of Salmonella units which move from carcass to carcass (Table 1; no. 11).

\section{Input data availability}

For the present study most of the input data were obtained from literature or advisory services (Table 1). No information was available about how many farrowing farms selling their pigs to one, two or three fatteners, the respective sow herd prevalence, the probability of an effective contact at transport and lairage and the proportion of Salmonella units remaining at lairage (Table 1; no. 2, 5, 6 and 8). Hence, information was acquired by discussion with experts.

\section{Number of replications}

For the executed appraisal, the decision on how many replications were needed to obtain sufficient model output, was based on the variance which would appear in the output data due to a certain number of replications. First, the model was run with default, minimum, and maximum values 200 times each (Table 1). Based on these 200 replications, 30 packages of 5 , $10,20,30, \ldots 100$ replications were randomly sampled with replacement. This bootstrapping was executed for minimum, maximum and default values. The variance of every package was calculated. Afterwards, the standard deviation of every package size, consisting of 30 variances each, was also calculated. Figure 3 shows the falling trend of standard deviation with increasing package size. Finally, the package size with 30 replications was considered as sufficient. There was a relatively sharp decline for the maximum graph up to 20 replications and for the default graph up to 30 replications. The further trend slowed down in decline. This indicates that the variance was no longer influenced by the number of replications.

'Default, minimum, and maximum values represent the three factor levels of the Placket-Burman design explained later. 


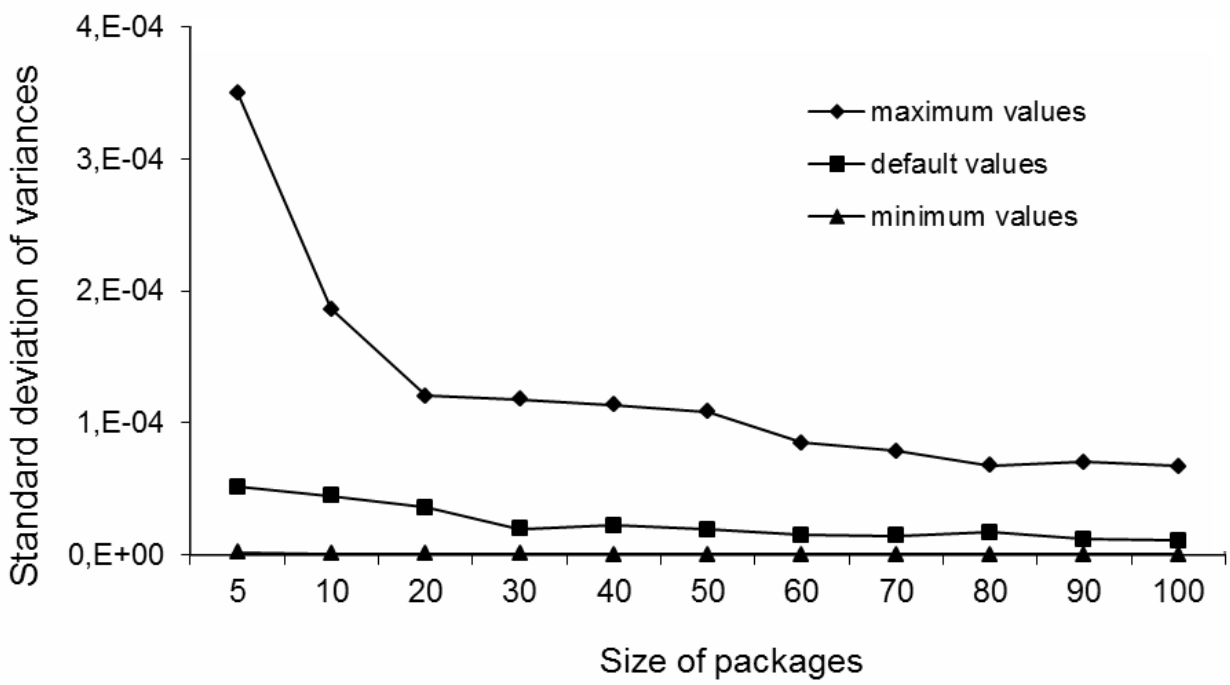

Figure 3

Standard deviation of variances related to the size of packages - obtained via bootstrapping method

\section{Sensitivity analysis}

A sensitivity analysis was performed to evaluate the model. A Plackett-Burman design (P-B design) was used to identify the most important input factors and to estimate their impact on the allocation of health states. Originally, P-B designs are two-level fractional factorial designs of resolution III for studying up to $\mathrm{k}=\mathrm{N}-1$ components in N runs (Montgomery 2005). To compare three factor levels in the analysis, the basic P-B design, considering maximum $(+)$ and default (0) values, had to be reflected. Therefore, the maximum values were replaced with the minimum (-) values (Figure 4a and 4b) (Vanaja \& Shobha Rani 2007).

In general, resolution III designs confound the main effects with two-factor interactions. To ensure that the main effects are not confounded with two-factor interactions, these designs have to be reversed (Barrentine 1996; Montgomery 2005). Hence, the basic and reflected designs were carried out again but extreme and default values were switched (Figure $4 \mathrm{c}$ and 4d). This full fold-over technique broke the alias links between all the main effects and their two-factor interactions and resulted in a design of resolution IV (Montgomery 2005). All together, 64 assemblies (four designs with 16 assemblies each) were performed considering the default, minimum and maximum values of the twelve factors described in Table 1. Default values of the probabilities were increased and decreased by $25 \%$ to obtain minimum and maximum values, respectively. Concerning proportions, minor parts were increased by $25 \%$ whereas the major part was decreased accordingly. For the Weibull distribution, the standard deviation was subtracted and added to the default value. Output data of the P-B design was analysed using the MIXED procedure of the SAS program package Version 9.1 (SAS Institute Inc., Cary, NC, USA). Linear models were fitted for the four health states, the transmission paths of infection and for the carcass contamination as well as for the loss of Salmonella due to careful evisceration (Figure 5). For every health state six models were formulated to analyse the variances over the course of time (Block A of Figure 5). To analyse Salmonella 
transmission paths, seven models were formulated (Block B of Figure 5 ) and for the loss of bacteria and the carcass contamination one model each was added (Block C of Figure 5). All 33 linear models considered the same twelve explanatory variables which represented the input factors of the simulation model. Analyses were done via F-Test, least square means and confidence limits. Corresponding to the P-B designs, the analysis had to consider three levels for every input factor. To adjust for these multiple comparisons, the $P$-values and confidence limits were accomplished with the Bonferroni adjustment.

a) Basic design

\begin{tabular}{rr|ccccc}
\hline \multirow{2}{*}{ Assembly } & \multicolumn{5}{|c}{ Input factors } \\
\cline { 3 - 6 } & 1 & 2 & 3 & $\ldots$ & 12 \\
\hline 1 & + & 0 & 0 & & 0 \\
2 & + & + & 0 & & + \\
3 & + & + & + & & 0 \\
$\vdots$ & & & & & \\
16 & 0 & 0 & 0 & & 0 \\
\hline
\end{tabular}

c) Reversion of the basic design

\begin{tabular}{r|c|cccc}
\hline \multicolumn{2}{c|}{ Assembly } & \multicolumn{5}{|c}{ Input factors } \\
\cline { 2 - 6 } & 1 & 2 & 3 & $\ldots$ & 12 \\
\hline 33 & 0 & + & + & & + \\
34 & 0 & 0 & + & & 0 \\
35 & 0 & 0 & 0 & & + \\
$\vdots$ & & & & & \\
48 & + & + & + & & + \\
\hline
\end{tabular}

b) Reflected design

\begin{tabular}{rr|rrrrr}
\hline \multirow{2}{*}{ Assembly } & \multicolumn{5}{|c}{ Input factors } \\
\cline { 3 - 6 } & & 1 & 2 & 3 & $\ldots$ & 12 \\
\hline 17 & - & 0 & 0 & & 0 \\
18 & - & - & 0 & & - \\
19 & - & - & - & & 0 \\
$\vdots$ & & & & & \\
32 & 0 & 0 & 0 & & 0 \\
\hline
\end{tabular}

d) Reversion of the reflected design

\begin{tabular}{rr|ccccc}
\hline \multirow{2}{*}{ Assembly } & \multicolumn{5}{|c}{ Input factors } \\
\cline { 3 - 6 } & & 1 & 2 & 3 & $\ldots$ & 12 \\
\hline 49 & 0 & - & - & & - \\
50 & 0 & 0 & - & & 0 \\
51 & 0 & 0 & 0 & & - \\
$\vdots$ & & & & & \\
64 & - & - & - & & - \\
\hline
\end{tabular}

Figure 4

Basic, reflected and reversed Plackett-Burman designs

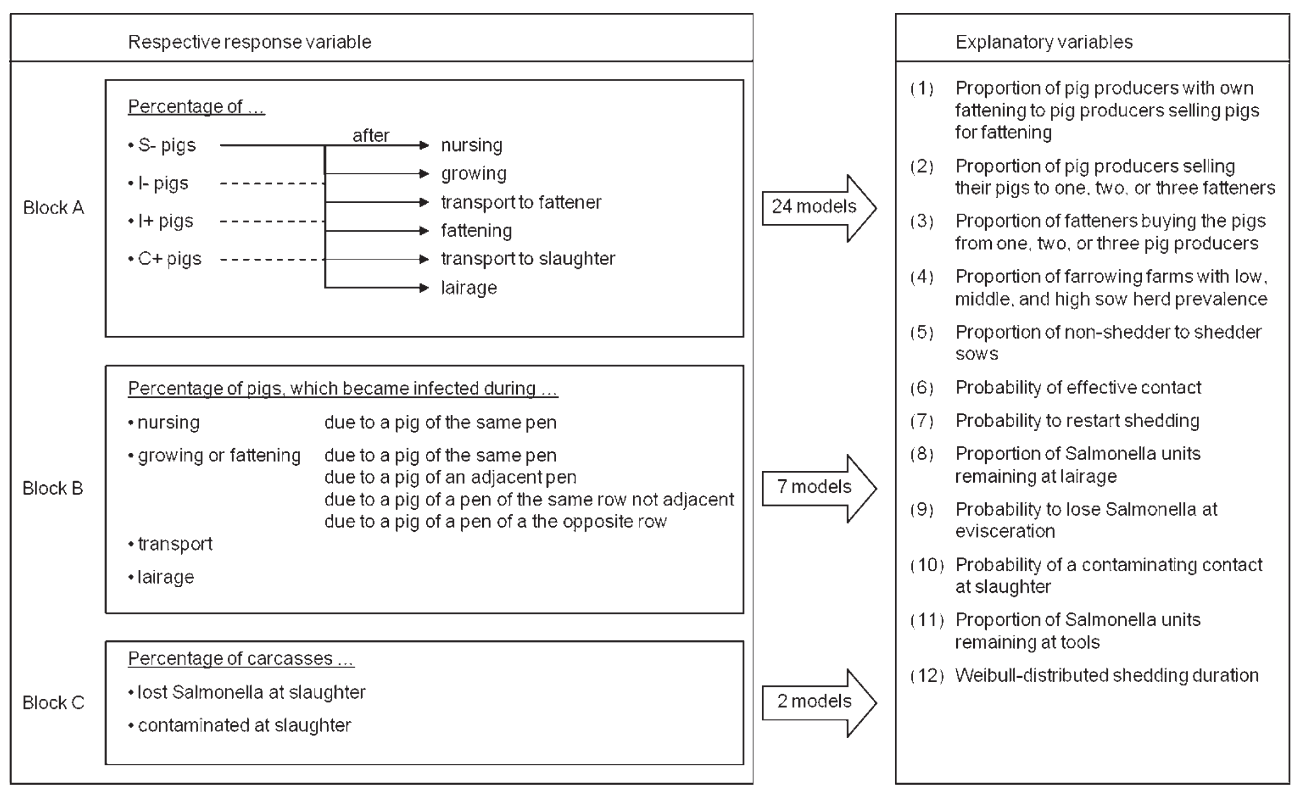

Figure 5

Outline of the formulated models for sensitivity analysis 


\section{Results}

First analyses considered only the assemblies 16, 48 and 64 of the P-B design, to receive an impression of the prevalence generated by the model. These three assemblies described the model output if all input factors were set to default, maximum and minimum values, respectively. After nursing, the percentages of infected piglets were very similar, but differences in prevalence increased in the course of time.

- Minimum values: prevalence after nursing: $0.04 \%$ (assembly 64)

- Default values: (assembly 16)

- Maximum values: $\quad$ prevalence after nursing: 0.14\% after lairage: $11.95 \%$ (assembly 48)

The proportion of infected pigs which lost Salmonella due to evisceration represented the given probabilities of about $56 \%$ (minimum values), $75 \%$ (default values) and $94 \%$ (maximum values). The carcass contamination never exceeded $2.32 \%$. (Results not presented.)

Further analyses were based on all 64 assemblies. The significance of the input factors for the response variables are shown in Table 2 . The presentation of the results for all 33 analyses would be too expansive. Hence, the presentation of the health state models is limited to the susceptible pigs (S-) (Table 2; Block A). This group represents exactly the opposite to the infected pigs $((\mathrm{I}-)+(\mathrm{I}+)+(\mathrm{C}+)$. Hence, factors with significant impact on the percentage of susceptible pigs influenced the amount of infected pigs, respectively. Factors 4-6 in Table 2 show that from nursing to slaughter the »Proportion of farrowing farms with low, middle, and high sow herd prevalence«, the »Proportion of non-shedder to shedder sows", as well as the »Probability of effective contact« have a significant impact on the percentage of susceptible pigs (Table 2; Block A). While the first two factors determined the amount of Salmonella units brought to model, the probability of effective contact effected the transmission dynamic of the present bacteria. Due to an average shedding duration of four weeks, Factor No. 12 did not become significant until growing. Even if pigs became infected at the week of birth, shedding duration probably exceeded the nursing time. Subsequently, the probability to restart shedding (no. 7) cannot be significant prior to that. Pigs have to finish shedding before they can restart. The factor »Proportion of Salmonella units remaining at lairage» (no.8) became significant at lairage.

The similarities between the significant factors for the percentage of susceptible pigs (Table 2; Block A) and the transmission paths (Table 2; Block B) were reasoned. The transmission paths were determined by effective contacts, which decreased the number of susceptible pigs.

Block $\mathrm{C}$ of Table 2 shows the significant input factors for the percentages of pigs which lost Salmonella at evisceration or became contaminated during slaughter process, respectively. Note that all pigs were considered to estimate the percentage of pigs which lost Salmonella; even susceptible pigs. Hence, additionally to the »Probability to lose Salmonella at evisceration« (no. 9) also factors influencing the percentage of infected pigs were significant. The same applies to the percentage of contaminated carcasses. Next to the »Probability of contaminating contact at slaughterhouse (no. 10) and the »Proportion of Salmonella units remaining at tools« (no.11), all factor groups influencing the amount of infected pigs were significant. 


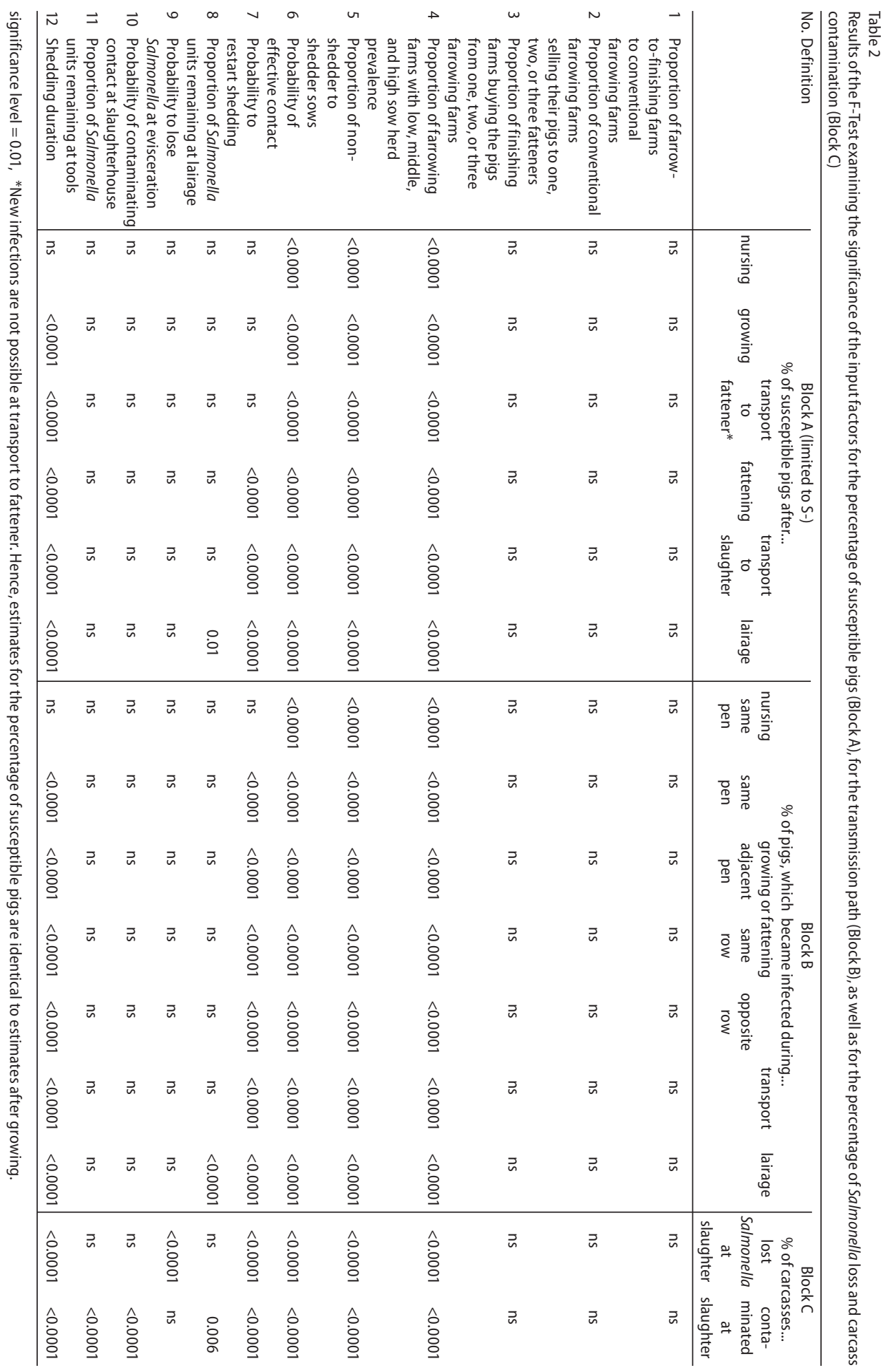


To illustrate the progress of infection, Figure 6 shows the least square means and their confidence limits for the percentage of susceptible pigs depending on factor groups 4-6 and 12. The most effective contacts happened during fattening where pigs spent most of their lives (Figure 6a). Figure 6b shows the importance of the sow herd prevalences at pig producing stage. Comparing the deviations from the default values, it became clear that the decrease in susceptible pigs caused by the maximum values exceeded the increasing effect of the minimum values. The same applied to the proportion of non-shedder to shedder sows (Figure $6 \mathrm{c}$ ). In contrast, the relation between shedding duration and percentage of susceptible pigs was linear. Increasing and decreasing effects balanced each other out (Figure $6 \mathrm{~d})$.The influence of transport-stress on the shedding reactivation is shown in Figure 7. The number of seropositive, shedding pigs (I+) increased especially at transport to slaughter, which increased the probability of a susceptible pig becoming infected.

(a) Probability of effective contact

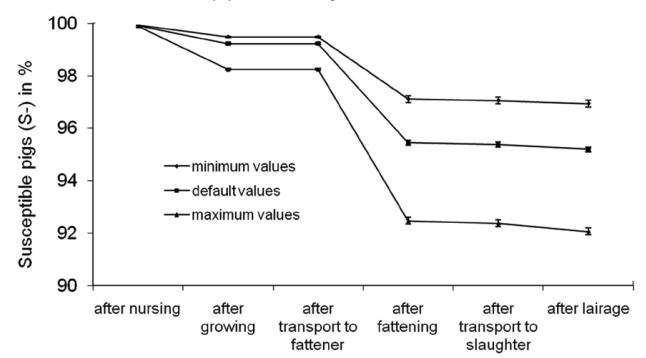

(c) Proportion of non-shedder to shedder sows

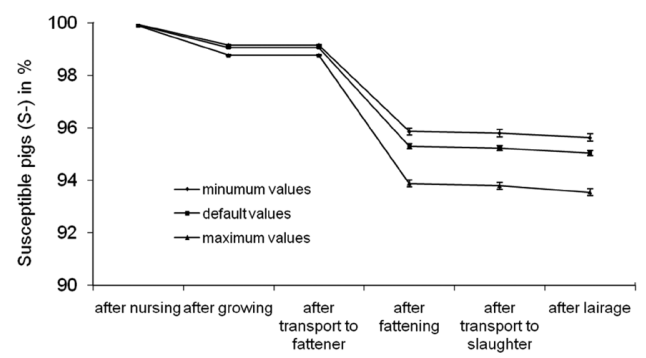

(b) Proportion of farrowing farms with low, middle

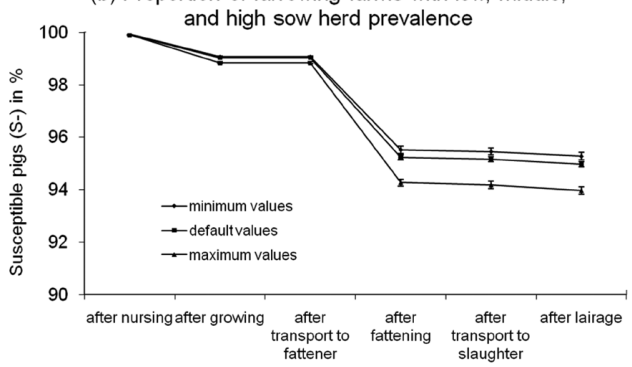

(d) Shedding duration

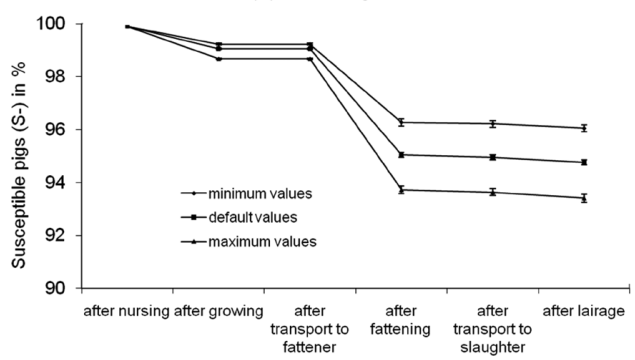

Figure 6

Least square means and confidence limits of the percentage of susceptible pigs depending on (a) the probability of effective contact, (b) proportion of farrowing farms with low, middle, and high sow herd prevalence, (c) the proportion of non-shedder to shedder sows, and (d) the shedding duration

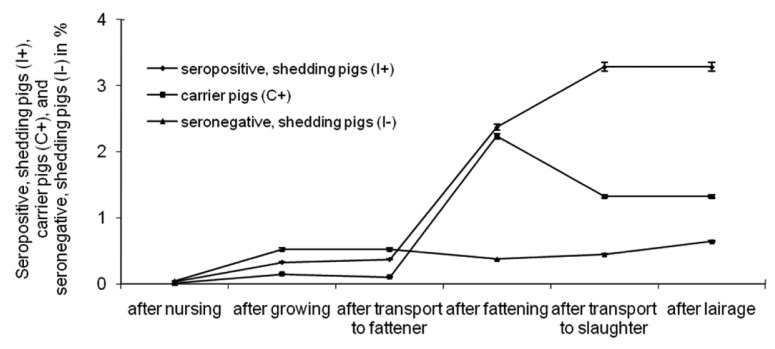

Figure 7

Least square means and confidence limits of the percentage of infectious, seronegative pigs, infectious, seropositive pigs, and carrier pigs depending on the probability to restart shedding (default value) 


\section{Discussion}

\section{Transition model}

The transition model considered both farm level and slaughter stage. The consideration of the four pig's health states was adequate for farm level. Until transport to slaughter, neglecting of the latent period between Salmonella ingestion and bacteria shedding was considered to be unproblematic since duration fell below the considered time step of one week. But Salmonella ingestion at transport to slaughter resulted immediately in the shedding of bacteria since an intermediate health state was missing. Hence, the chance of a susceptible pig having become infected at lairage might have been overestimated. But infection at lairage represented about $5 \%$ in the maximum case. Overestimation was neither of little consequence for carcass contamination. The passive immunity of newborn piglets was not considered either. Lurette et al. (2008) described the maternal protective factor as one of the most influential parameters on Salmonella prevalence in delivered pigs but precise information about this effect is missing. In support, Nollet et al. (2005) could not prove the direct transmission of Salmonella from the sows to their piglets at farrowing barn, which confirms passive immunity. But they demonstrated similarities between the isolates found in the sows and those found during growing, finishing and at slaughter. Hence, even if passive immunity of newborn piglets exists, the sow may play a significant role in the indirect transmission of Salmonella to growing and finishing pigs (Nollet et al. 2005). In the present study, not even $0.15 \%$ of the piglets had effective contact during nursing. The differences between minimum, default, and maximum values were very small after nursing but increased in the course of time. In the maximum scenario, slaughter pig prevalences of $12 \%$ were reached. This prevalence seemed to be very high, considering that the sows were the only initial infection source in the model presented. But it has to be considered that within the model no infection remained undetected, not even prior to seroconversion. In contrast, empirical prevalences based on antibody detection may never represent all infected pigs at a particular time (Battenberg 2007). Nevertheless, the obtained slaughter pig prevalences emphasize the impact of the sow for Salmonella entrance and the subsequent transmission. The transmission dynamic depends extremely on how many piglets come into contact with Salmonella during nursing.

\section{Number of replications}

The determination of the number of replications is important and difficult at once. Vynnycky \& White (2010) emphasise that the number of replications is usually limited by the realisation time. If several models are to be compared, the determinations rely on the number of replications which are required to analyse statistical differences between the models (Chung 2004). But the present study did not compare models. Instead, the number of replications was of concern, which ensured that enough random numbers were used to represent the underlying distribution.

Usually, if this number of replications is obtained, the variance of the output does not decrease due to additional replications. Variations are casual and balance each other. This point was determined to be reached with 30 replications. Some might prefer 80 replications 
due to further flattening within the trend of the maximum graph, but because of the already small level of standard deviation the additional realisation time seemed to be unjustified.

\section{Sensitivity analysis}

Due to the relative high number of input factors a screening design was appropriate to identify the most important factors and evaluate the model based on their estimated effects. P-B designs are screening designs, which estimate unbiased main effects in the smallest design possible (Vanaja \& Shobha Rani 2007). The limited number of runs minimised the realisation time and offered the comparison of three levels instead of two. Thus, it could be shown that the relationship between several factors and response variable is non-linear. An increase in the probability of effective contact, a higher proportion of pig producers with middle and high sow herd prevalence, or more shedder sows decreased the percentage of susceptible pigs much more as could have been increased by the minimum values (Figure 6).

The problem that the main effects are confounded with two-factor interactions in a P-B design was solved by reversing the designs. The resulting fold-over pairs were of resolution IV, which did not confound the main effects and two-factor interactions (Box et al. 1978, Montgomery 2005). But in contrast to traditional designs of resolution IV, a P-B design does not allow the estimation of interactions between factors (Vanaja \& Shobha Rani 2007). Hence, it cannot be ruled out that there are significant relationships between factors which remained undetected.

The estimated main effects of the model met expectations. To avoid that biologically nonrelevant differences become significant, the significance level for the F-Test was reduced to $1 \%$. In simulation designs with large sample sizes smallest differences become significant. According to Ivanek et al. (2004) the probability of effective contact (no. 6), the probability of restarting shedding (no. 7), as well as the shedding duration (no. 12) were proven to be significant. Hill et al. (2008) point out that the most effective control strategies are those that reduce the probability of effective contact between pens. The relevance of the proportion of farrowing farms with low, middle, and high sow herd prevalence (no. 4) as well as the importance of the proportion of non-shedder to shedder sows (no. 5) cannot be compared exactly with other studies, since previous models known by the authors did not consider the sow as initial source of Salmonella infection in detail. But van der Gaag et al. (2004) proved that a higher starting prevalence at farrowing stage results in more infected animals. Furthermore, Ivanek et al. (2004) state that Salmonella transmission is influenced by the prevalence among weaners.

For the contamination and loss of bacteria at slaughter, detailed information and simulation studies are rare. Van der Gaag et al. (2004) describe the slaughterhouse as one of the most important stages in the supply chain to reduce the prevalence of Salmonellacontaminated carcasses. Accordingly, the present study showed that a lot of infected pigs lose bacteria due to careful evisceration. But these pigs remain seropositive and increase the herd prevalence even if there is no risk of humans becoming infected. The herd prevalence determines the classification of farms within quality assurance systems and is therefore of paramount importance, especially for the fatteners. The present study showed that efforts to decrease the herd prevalence should be focused on Salmonella entry and the transmission 
via effective contacts between pigs. A conceivable measure to reduce Salmonella entry via farrowing sows might be the vaccination of sows and piglets as well the removal of faeces from the pen. Furthermore, the susceptibility of pigs to present bacteria should be reduced. Common recommendations are the acidification of feed or water, rodent control, intensive cleaning and disinfection, et cetera. Subsequent studies will expand the presented model to horizontal entry of Salmonella and will analyse the effectiveness of several prevalencereducing strategies.

\section{References}

Bailey N (1975) The mathematical theory of infectious diseases. Griffin, London, UK

Barrentine LB (1996) Illustration of confounding in Plackett-Burman designs. Quality Engineering 9, 11-20

Battenberg L (2007) [Investigation of the ways of Salmonella invasion into selected Bavarian pig farms]. Diss. Veterinary Faculty, Ludwig-Maximilians-University, Munich, Germany [in German]

BfR (2005) Salmonella in pork - still a risk. Press release of the Federal Institute of Risk Assesment (BfR), http://www.bfr.bund.de/cd/6075 [last accessed 18.01.2012]

Box GEP, Hunter WG, Hunter JS (1978) Statistics for Experimenters - An Introduction to Design, Data Analysis, and Model Building. John Wiley \& Sons, New York, USA

Chung CA (2004) Simulation modeling handbook: a practical approach. CRC Press, Florida, USA

EFSA (2008) Report of the Task Force on Zoonoses Data Collection on the analysis of the baseline survey on the prevalence of Salmonella in slaughter pigs, in the EU, 2006-2007 Part A: Salmonella prevalence estimates. The EFSA Journal European Food Safety Authority (EFSA), 1-111

Hill AA, Snary, EL, Arnold ME, Alban L, Cook AJC (2008) Dynamics of Salmonella transmission on a British pig grower-finisher farm: a stochastic model. Epidemiology and Infection 136, 320-333

Ivanek R, Snary EL, Cook AJC, Grohn YT (2004) A mathematical model for the transmission of Salmonella Typhimurium within a grower-finisher pig herd in Great Britain. Journal of Food Protection 67, 2403-2409

Krieter J (2004) Evaluation of Salmonella surveillance in pigs using a stochastic simulation model. Arch Tierz 47, 337-349

Lurette A, Belloc C, Touzeau S, Hoch T, Ezanno P, Seegers H, Fourichon C (2008) Modelling Salmonella spread within a farrow-to-finish pig herd. Vet Res 39, 49

Montgomery DC (2005) Design and Analysis of Experiments. Wiley, Hoboken, NJ, USA

NAG (2001) The NAG C Library Manual - Mark 7. Numerical Algorithms Group Ltd, Oxford, UK

Nollet N, Houf K, Dewulf J, Duchateau L, De Zutter L, De Kruif A, Maes D (2005) Distribution of Salmonella strains in farrow-to-finish pig herds: A longitudinal study. J Food Prot 68, 2012-2021

Rubel F (2005) Process Models in Veterinary Epidemiology. Department of Natural Sciences Vienna [in German]

Struck J (2009) [Hunt for Salmonella will be intensified]. Agrarzeitung 48, 6 [in German]

van der Gaag MA, Vos F, Saatkamp HW, van Boven M, van Beek P, Huirne RBM (2004) A state-transition simulation model for the spread of Salmonella in the pork supply chain. EJOR 156, 782-798

Vanaja K, Shobha Rani RH (2007) Design of Experiments: Concept and Applications of Plackett Burman design. Clin Res Regul Aff 24, 1-23

Vynnycky E, White RG (2010) An Introduction to Infectious Disease Modelling. Oxford University Press, New York, USA

Wehebrink T, Kemper N, Krieter J (2007) Simulation study on the epidemiology of Salmonella spp. in the pork supply chain. Campylobacter spp., Yersinia spp. and Salmonella spp. as Zoonotic Pathogens in Pig Production. Institute of Animal Breeding and Husbandry, Christian-Albrechts-University, Kiel, Germany

WHO (2007) Food safety and foodborne illness. Fact sheet N²37. World Health Organization, http://www. who.int/mediacentre/factsheets/fs237/en/ [last accessed 9.8.2010] 
WHO (2010) The European health report 2009: health and health systems. World Health Organization, http:// www.euro.who.int/__data/assets/pdf_file/0009/82386/E93103.pdf [last accessed 9.8.2010]

ZDS (2009) >>Erzeugerring<< online database. http://www.erzeugerring.info [in German]

Received 28 May 2011, accepted 3 November 2011.

Corresponding author:

Stefanie Hotes

email: shotes@tierzucht.uni-kiel.de

Institute of Animal Breeding and Husbandry, Christian-Albrechts-Universität zu Kiel, Olshausenstraße 40, 24098 Kiel, Germany 